\title{
FACTORIZATION AND CONGRUENCE IN THE ARITHMETICS OF CAYLEY'S ALGEBRA
}

\author{
by P. J. C. LAMONT
}

(Received 7 December, 1989)

This paper treats factorization and congruence in the arithmetics of Cayley's algebra $C$. Results, proved by Rankin [10], on the number of factorizations of a given element in the nonmaximal arithmetics $H_{0}$ and $J_{0}$ are reviewed. Further, new results on congruence are established and are used to find the number of factorizations of a prescribed element in the remaining arithmetics, including the maximal arithmetics $J_{w}$. When computer factorizing a given element, the congruence results can be used as a heuristic device to prune the search tree.

Let $J$ be any arithmetic of Cayley's algebra $C$. If, for any two elements $\xi$, $\xi$ of an arithmetic $J$ of $C$, there exists an element $\eta$ of $J$ such that

$$
\zeta=\xi \eta
$$

$\zeta$ is said to be divisible by $\xi$ on the left in $J$ and $\xi$ is said to divide $\zeta$ on the left in $J$. Similarly we define $\zeta$ to be divisible by $\eta$ on the right in $J$ if there exists an $\xi$ of $J$ such that (1) holds. In both cases $\zeta$ is said to have the factorization $\xi \eta$ in $J$ and $\xi, \eta$ are called factors of $\zeta$ in $J$. We write

$$
\zeta=\xi \eta \text { in } J .
$$

For example, an element $\rho$ of norm 1 in $J$, henceforth called a unit of $J$, divides any element $\zeta$ of $J$ on the left and on the right in $J$. Also, clearly, a rational integer $m$ divides an element $\zeta$ of $J$ on the right in $J$ if and only if $m$ divides $\zeta$ on the left in $J$.

An element $\zeta$ of $J$ is said to be a Cayley prime for $J$ if, for all factorizations $\xi \eta$ of $\zeta$ in $J$, either $N \xi=1$ or $N \eta=1$. For example, suppose that $\zeta$ has norm a rational prime $p$, say. Then it follows, since $N \zeta=N \xi N \eta=p$, that either $\xi$ or $\eta$ has norm 1. In this case, $\zeta$ is a Cayley prime.

If, for given quaternions $\alpha, \beta, \gamma$,

$$
\alpha=\beta \gamma
$$

it follows, by the associative law of multiplication for quaternions, that for any quaternion $\delta$

$$
\alpha=(\beta \delta)\left(\delta^{-1} \gamma\right) .
$$

Thus if $\beta$ divides $\alpha$ on the left in a quaternion arithmetic $H^{\prime}$ of $C$, it follows that for any unit $\delta$ belonging to $H^{\prime}, \beta \delta$ divides $\alpha$ on the left in $H^{\prime}$.

If $\zeta, \xi, \eta$ are Cayley numbers and $\xi=\xi \eta$ the relation

$$
\zeta=(\xi \delta)\left(\delta^{-1} \eta\right)
$$

in general only holds when $\delta$ is a nonzero real number.

Suppose that, for $N \xi=N \xi^{\prime}$ and $N \eta=N \eta^{\prime}$,

$$
\zeta=\xi \eta=\xi^{\prime} \eta^{\prime}
$$

are two different factorizations in arithmetic $J$ of an element $\zeta$ of $J$. Then, from [9], it follows that no explicit relation. corresponding to a principal isotopism of $C$ can exist 
between $(\xi, \eta)$ and $\left(\xi^{\prime}, \eta^{\prime}\right)$ other than

$$
\xi=-\xi^{\prime}, \quad \eta=-\eta^{\prime}
$$

unless $\zeta$ of $\zeta u$ is a quaternion for some unit $u$ of $C$ or $\zeta$ is of some other special form.

We shall see that the factors of a given element of $J_{w}$ can be characterized up to a factor of \pm 1 by considering congruence modulo 2 in $J_{w}$.

For any rational integer $m>0$ and Cayley integers $\xi, \eta$ of $J_{w}$, we define $\xi$ to be congruent to $\eta$ modulo $m$ in $J_{w}$ if $\xi-\eta$ is divisible by $m$ in $J_{w}$. We then write

$$
\xi \equiv \eta \quad \text { (modulo } m \text { in } J_{w} \text { ) }
$$

or, when no confusion can arise,

$$
\xi \equiv \eta \quad(\bmod m) .
$$

It is clear that for this definition congruence is well defined in the sense that it is an equivalence relation.

If

then

$$
\xi \equiv \xi_{1} \quad(\bmod m), \quad \eta \equiv \eta_{1} \quad(\bmod m)
$$

$$
\xi \eta \equiv \xi_{1} \eta_{1} \quad(\bmod m) .
$$

We only consider modulus $m$ for $m$ a rational integer.

We first prove

THEOREM 1. For any elements $\eta$ and $\eta^{\prime}$ of $J_{w}$ such that

$$
\eta \equiv \eta^{\prime} \quad\left(\operatorname{modulo} 2 \text { in } J_{w}\right)
$$

$N \eta$ and $N \eta^{\prime}$ are either both even or both odd rational integers.

We have $\eta=\eta^{\prime}+2 \zeta$ where $\zeta$ is an element of $J_{w}$. Therefore,

$$
N \eta=N \eta^{\prime}+4 N \zeta+2 R\left(2 \eta^{\prime} \bar{\zeta}\right) .
$$

But $n^{\prime} \xi$ is an element of $J_{w}$. Hence $R\left(2 \eta^{\prime} \bar{\xi}\right)$ must be an integer. The result follows.

Also we have

THEOREM 2. Any element $\zeta$ of maximal arithmetic $J_{w}$ is congruent modulo 2 in $J_{w}$ to an element $\tau$ of an arithmetic $J$, containing $J_{0}$, of $C$ if and only if $\zeta$ is itself an element of $J$.

We have

$$
\zeta \equiv \tau \quad\left(\text { modulo } 2 \text { in } J_{w}\right)
$$

Thus

$$
\zeta=\tau+2 \alpha
$$

where $\alpha$ is an element of $J_{w}$. Thus $2 \alpha$ is contained in $J_{0}$. Hence $\zeta$ belongs to $J$ if and only if $\tau$ belongs to $J$.

We now prove the more difficult

THEOREM 3. Any element $\xi$ of odd norm of a maximal arithmetic $J_{w}$ of Cayley's algebra $C$ is congruent modulo 2 in $J_{w}$ to an element, unique apart from sign, of norm 1 of $J_{w}$. 
Let $\xi$ be any given element of odd norm in $J_{w}$. Then, from the definition of $J_{w}$, it follows, since $\xi$ has odd norm, that, with the notation of [3],

$$
\xi=\alpha_{0}+\delta_{1} \xi_{(w)}
$$

where $\alpha_{0}$ is an element of $J_{0}, \xi_{(w)}$ equals $\xi_{u}, \xi_{u}^{*}, \xi_{w, v}$ or $\xi_{w, v}^{*}$ and $u$ is a basic unit assigned to an associative triad containing $w$, and $v$ is a basic unit assigned to a triad not containing $w$.

Suppose that $\delta_{1}=0$. Then $\xi=\alpha_{0}$ and

$$
\alpha_{0} \equiv \sum_{s=0}^{7} a_{s} i_{s} \quad(\bmod 2)
$$

where each $a_{s}$ is 0 or 1 . Since $N \alpha_{0}$ is odd, the numer $r$ of coefficients for which $a_{s}=1$ $(0 \leq s \leq 7)$ must be odd.

If $r=1$ the result follows.

If $r=3$ we have

$$
\alpha_{0} \equiv i_{s_{1}}+i_{s_{2}}+i_{s_{3}} \quad(\bmod 2) .
$$

It is easy to prove that there exists a basic unit $i_{t}$ of $C$ for which $\frac{1}{2}\left(i_{t}+i_{s_{1}}+i_{s_{2}}+i_{s_{3}}\right)$ is contained in $J_{w}$. But for any such set $\left(u_{1}, u_{2}, u_{3}, u_{4}\right)$

Thus in this case

$$
\sum_{l=1}^{4} u_{l} \equiv 0 \quad(\bmod 2)
$$

If $r=5$ we have, since

$$
\alpha_{0} \equiv i_{t} \quad(\bmod 2) .
$$

$$
\sum_{i=0}^{7} i_{l} \equiv 0 \quad(\bmod 2),
$$

$\alpha_{0}$ congruent modulo 2 in $J_{w}$ to the sum of three different basic units of $C$. The case $r=5$ has thus been reduced to the case $r=3$.

Similarly the result follows if $r=7$.

If $\delta_{1}=1$, we have

$$
\xi=\alpha_{0}+\xi_{(w)} .
$$

From above we see that if $N \alpha_{0}$ is odd, $\alpha_{0}$ is congruent modulo 2 in $J_{w}$ to a basic unit $i_{t}$, say, of $J_{0}$. Now write

$$
\xi_{(w)}=\frac{1}{2} \sum_{l=1}^{4} w_{l}
$$

where $w_{s}(1 \leq s \leq 8)$ are the eight basic units of $J_{0}$.

Then we have $i_{t}=w_{1}$, for some $l_{1}\left(1 \leq l_{1} \leq 4\right)$ for if not $\xi$ is congruent modulo 2 in $J_{w}$ to an element of even norm of $J_{w}$ which cannot be true. Thus

$$
\xi \equiv \frac{1}{2}\left(-w_{l_{1}}+w_{l_{2}}+w_{l_{3}}+w_{l_{4}}\right) \quad(\bmod 2)
$$

and the result follows.

Now suppose that $N \alpha_{0}$ is even. Then

$$
\alpha_{0} \equiv \sum_{s=0}^{7} a_{s} i_{s} \quad(\bmod 2)
$$

where each $a_{s}=0$ or 1 . Since $N \alpha_{0}$ is even the number $r$ of coefficients $a_{s}=1$ must be even. 
If $r=0$ or 8 the result follows at once.

If $r=2$ or 6 we have

where as before

$$
\xi \equiv i_{s_{1}}+i_{s_{2}}+\xi_{(w)}(\bmod 2)
$$

$$
\xi_{(w)}=\frac{1}{2} \sum_{l=1}^{4} w_{l}
$$

and the $w$ 's form a set $D$, say, of four basic units of $C$. Precisely one of $i_{s_{1}}$ and $i_{s_{2}}$ cannot occur in $D$, for $\xi$ must be congruent modulo 2 in $J_{w}$ to an element of odd norm. If neither $i_{s_{1}}$ nor $i_{s_{2}}$ occurs in $D$ the result follows as before. We now suppose that both $i_{s_{1}}$ and $i_{s_{2}}$ occur in the set $D$. In this case it is easy to prove that there exist two elements $w_{s_{1}}, w_{s_{2}}$ of $D$ for which

is a contained in $J_{w}$.

$$
\frac{1}{2}\left(i_{s_{1}}+i_{s_{2}}+w_{s_{1}}+w_{s_{2}}\right)
$$

We deduce that

Hence

$$
i_{s_{1}}+i_{s_{2}} \equiv w_{s_{1}}+w_{s_{2}} \quad(\bmod 2)
$$

$$
\xi \equiv \frac{1}{2}\left(-w_{s_{1}}-w_{s_{2}}+w_{s_{3}}+w_{s_{4}}\right) \quad(\bmod 2) \text {. }
$$

Finally we suppose that $r=4$. Then

$$
\xi \equiv i_{s_{1}}+i_{s_{2}}+i_{s_{3}}+i_{s_{4}}+\frac{1}{2}\left(w_{1}+w_{2}+w_{3}+w_{4}\right) \quad(\bmod 2)
$$

Clearly the sets $\left(i_{s_{1}}, i_{s_{2}}, i_{s_{3}}, i_{s_{4}}\right)$ and $\left(w_{1}, w_{2}, w_{3}, w_{4}\right)$ must have an even number of elements in common for otherwise $\xi$ is congruent modulo 2 in $J_{w}$ to an element of even norm. If the sets have no element in common or four elements in common the results is immediate. If they have precisely two elements in common the argument reduces to the previous case.

Thus for any element $\xi$ contained in $J_{w}$

$$
\xi \equiv \rho \quad\left(\text { modulo } 2 \text { in } J_{w}\right)
$$

where $\rho$ is an element of norm 1 of $J_{w}$. If also

$$
\xi \equiv \rho^{\prime} \quad\left(\text { modulo } 2 \text { in } J_{w}\right)
$$

where $N \rho^{\prime}=1$ it follows that $\rho= \pm \rho^{\prime}$. Theorem 3 has thus been proved.

As an example we consider the element

$$
\xi=1+i_{1}+\frac{1}{2}\left(i_{4}+i_{5}+i_{6}-i_{7}\right) .
$$

Since the characteristic unit $[3] x(\xi)=1$ and

$$
\xi=1+i_{1}+\frac{1}{2}\left(1+i_{1}+i_{2}+i_{3}\right) i_{4}=\alpha_{0}+\xi_{(w)}
$$

where $\alpha_{0}=1+i_{1}$ and $\xi_{(w)}=\xi_{i_{4}}^{*}$, we can take $w=i_{1}, i_{2}$ or $i_{3}$. In $J_{i_{3}}$ we have

$$
\beta=1-i_{1}+i_{4}+i_{6} \equiv 0(\bmod 2)
$$

since $x\left(\frac{1}{2} \beta\right)=i_{3}$. Thus in $J_{i_{3}}$

while in $J_{i}$, we have

$$
\begin{aligned}
\xi & \equiv-i_{4}-i_{6}+\frac{1}{2}\left(i_{4}+i_{5}+i_{6}+i_{7}\right) \quad(\bmod 2) \\
& \equiv \frac{1}{2}\left(-i_{4}+i_{5}-i_{6}+i_{7}\right) \quad(\bmod 2)
\end{aligned}
$$

$$
\xi \equiv \frac{1}{2}\left(+i_{4}+i_{5}-i_{6}-i_{7}\right) \quad(\bmod 2) .
$$

Next we deduce from Theorem 3 
TheOREM 4. Any element $\eta$ of even norm of a maximal arithmetic $J_{w}$ of Cayley's algebra $C$ is congruent modulo 2 in $J_{w}$ to the sum of two elcments of $J_{w}$ of norm 1 or to zero.

This follows since any such element $\eta$ can be written as $\eta_{1}+\eta_{2}$ where $\eta_{1}$ and $\eta_{2}$ are linear combinations of disjoint defining sets of units for $J_{w}$ and are such that $N \eta_{1}$ and $N \eta_{2}$ are odd.

Theorems 3 and 4 are used to characterize and count the number of distinct factors in maximal arithmetic $J_{w}$ of a given element $\zeta$ of $J_{w}$. However, we must first relate the number of representations of odd rational integer $m n$ as $\sum_{s=0}^{7} z_{s}^{2}$, where $\sum_{s=0}^{7} z_{s} i_{s}$ is an element of $J_{w}$, to the number of representations of rational integers $m$ and $n$ of this form.

We define $r_{h}(m)$ to be the number of different representations of $m$ as $\sum_{s=0}^{7} x_{s}^{2}$ where $\sum_{s=0}^{7} x_{s} i_{s}$ is contained in fixed arithmetic $J_{h}$ of $C$ for $h$ an integer between 0 and 7 or one of the seven basic units of $C$ other than 1. Further we write $r_{0}(m)=r(m)$. Clearly,

$$
r_{s}(m)=r_{t}(m) \text { and } r_{i_{s}}(m)=r_{i_{t}}(m)
$$

for $s$ and $t$ between 1 and 7. For example, $r(1)=16$, and $r_{s}(1)=48$ and $r_{i_{s}}=240$ for $1 \leq s \leq 7$.

We state without proof some results on the number of distinct representations of a rational integer as the norm of an element of any fixed arithmetic of $C$ containing the eight basic units of $C$.

THEOREM 5.

(i) For an odd rational integer

$$
r_{h}(m) r(1)=r(m) r_{h}(1) .
$$

(ii) For $m, n$ odd rational integers such that $(m, n)=1$

$$
r_{h}(m) r_{h}(n)=r_{h}(m n) r_{h}(1) \text {. }
$$

(iii) For $p$ a rational prime and integer $t>0$

and

$$
r_{h}(p)=r_{h}(1)\left(1+p^{3}\right)
$$

$$
r_{h}(1) r_{h}\left(p^{t+1}\right)=r_{h}(p) r_{h}\left(p^{t}\right)-r_{h}(1) p^{3} r_{h}\left(p^{t-1}\right) .
$$

(iv) For integer $t>0$

$$
r\left(2^{t}\right)=16\left\{\sum_{s=1}^{t} 2^{3 s}-1\right\}
$$

where in each case $h$ may take any one of the values $0,1, \ldots, 7, i_{1}, i_{2}, \ldots, i_{7}$. odd,

The results for $r(m)$ are given in Rankin's paper [10]. Then it follows that, for $m$

$$
r(4 m)=\frac{1}{16} r(4) r(m)=71 r(m) .
$$

Hence the number of representations of $4 m$ as a sum of eight squares of integers, four of 
which are odd, is $70 r(m)$. Thus, from Theorem 5(i), it follows that

and

$$
r_{i_{s}}(m)=15 r(m)
$$

$$
r_{s}(m)=3 r(m) \text { for } 1 \leq s \leq 7 \text {. }
$$

An independent proof of Theorem 5 can be given by means of the methods indicated by Rankin.

We now prove

THEOREM 6. Any element $\xi$ of maximal arithmetic $J_{w}$ of $C$ for which $N \xi=m n$ where $m, n$ are positive rational integers such that $(m, n)=1$ has precisely 240 different factorizations $\xi \eta$ in $J_{w}$ for which $N \xi=m$ and $N \eta=n$. For if

If $N \xi_{1}=m=N \xi_{2}$ and $\xi_{1} \neq \pm \xi_{2}$ then the absolute value of $R\left(\xi_{1}, \xi_{2}\right)$ is less than $m$.

we have

$$
\xi_{t}=\sum_{s=0}^{7} x_{t s} i_{s} \quad(t=1,2)
$$

$$
N\left(\xi_{1} \pm \xi_{2}\right)=\sum_{s=0}^{7}\left(x_{1 s} \pm x_{2 s}\right)^{2}<2 \sum_{s=0}^{7}\left(x_{1 s}^{2} \pm x_{2 s}^{2}\right)=4 m \text {. }
$$

Further, suppose that $\zeta$ is dividisible on the left by $\xi_{1}$ and $\xi_{2}$ in $J_{w}$ where $N \xi_{1}=N \xi_{2}=m$. Write

Then

$$
\zeta=\xi_{1} \eta_{1} \text { and } \zeta=\xi_{2} \eta_{2} \text {. }
$$

Similarly,

$$
\zeta \bar{\eta}_{2}=\left(\xi_{2} \eta_{2}\right) \bar{\eta}_{2}=\xi_{2} n \text {. }
$$

Thus

$$
\eta_{1} \bar{\zeta}=n \bar{\xi}_{1} \text {. }
$$

Hence,

$$
n^{2} \bar{\xi}_{1} \xi_{2}=\left(\eta_{1} \bar{\zeta}\right)\left(\zeta \bar{\eta}_{2}\right) \text {. }
$$

$$
n^{2} R\left(\bar{\xi}_{1} \xi_{2}\right)=N \xi R\left(\eta_{1} \bar{\eta}_{2}\right) .
$$

But $(m, n)=1$. Therefore,

Suppose further that

$$
R\left(\bar{\xi}_{1} \xi_{2}\right)=0 \text { or } \pm \frac{1}{2} m
$$

Then

$$
\xi_{1} \equiv \xi_{2} \quad\left(\bmod 2 \text { in } J_{w}\right) \text {. }
$$

$$
\bar{\xi}_{1} \xi_{2} \equiv 1 \quad\left(\bmod 2 \text { in } J_{w}\right) .
$$

Thus, by Theorem $2, \bar{\xi}_{1} \xi_{2}$ is contained in $J_{0}$. Hence

$$
R\left(\xi_{1} \xi_{2}\right)=0 \text {. }
$$

Now $N\left(\xi_{1} \xi_{2}\right)=m^{2}$ and $m$ is odd. Therefore, $\bar{\xi}_{1} \xi_{2}$ has one or five odd rational integral components. It is easy to show that any set of five basic units of $C$ contains a basic defining set of units for $J_{w}$. Thus the sum of any five such basic units is congruent modulo 2 in $J_{w}$ to one of the five units. Hence

for some $t,(1 \leq t \leq 7)$.

$$
\bar{\xi}_{1} \xi_{2} \equiv i_{t} \quad(\bmod 2)
$$


But (2) and (4) cannot both hold. Hence

$$
\xi_{1} \not \xi_{2} \quad(\bmod 2) \text {. }
$$

From (5), Theorem 3 and the fact that

$$
r_{w}(1)=240,
$$

it follows that any $\zeta$ of odd norm $m n$ where $(m, n)=1$ has at most 240 factorizations $\xi \eta$ in $J_{w}$ such that $N \xi=m, N \eta=n$.

Now suppose that there exists a $\zeta$ in $J_{w}$ of norm $m n$ with less than 240 such factorizations. For all such $\zeta$ for which $N \zeta=m n$ the number of factorizations of this form is given by $r_{w}(m) r_{w}(n)$.

We deduce that

$$
r_{w}(m) r_{w}(n)<240 \sum_{N \zeta=m n} 1=240 r_{w}(m n) .
$$

But this contradicts Theorem 5(ii) with $h=w$. This completes the proof of Theorem 6 .

Next we prove

THEOREM 7. Any element $\zeta$ of maximal arithmetic $J_{w}$ of $C$ for which $N \zeta=p^{l+1}$ where $p$ is an odd rational prime and $l>0$ has precisely

(i) $240\left(1+p^{3}\right)$ distinct factorizations $\xi \eta$ in $J_{w}$ for which $N \xi=p$ and $N \eta=p^{l}$ if $p$ divides $\zeta$ in $J_{w}$ or

(ii) 240 such factorizations if $p$ does not divide $\zeta$ in $J_{w}$.

Proof. (i) Suppose that $p$ divides $\zeta$ in $J_{w}$. Then $\zeta=p \zeta^{\prime}$ where $\zeta^{\prime}$ is contained in $J_{w}$. Let $\xi$ be any element of $J_{w}$ of norm $p$ and suppose that $\eta=\bar{\xi} \zeta^{\prime}$. Then $\eta$ is contained in $J_{w}$. Also

$$
\xi \eta=\xi\left(\xi \zeta^{\prime}\right)=p \xi^{\prime}=\xi .
$$

Thus $\zeta$ has as many distinct factorizations $\xi \eta$ with $N \xi=p$ and $N \eta=p^{l}$ as there are distinct elements of norm $p$ in $J_{w}$. The result follows from (iii) of Theorem 5.

(ii) Suppose now that $p$ does not divide $\zeta$ in $J_{w}$. Let $\zeta$ have distinct factorizations $\xi_{1} \eta_{1}$ and $\xi_{2} \eta_{2}$ in $J_{w}$ for which

$$
N \xi_{1}=N \xi_{2}=p \text { and } N \eta_{1}=N \eta_{2}=p^{l} .
$$

Suppose that

Then

$$
\xi_{1} \equiv \xi_{2} \quad\left(\bmod 2 \text { in } J_{w}\right) .
$$

$$
\xi_{1} \bar{\xi}_{2} \equiv 1 \quad\left(\bmod 2 \text { in } J_{w}\right) \text {. }
$$

Thus, by Theorem $2, \xi_{1} \xi_{2}$ is an element of $J_{0}$.

Now, we have

But,

$$
R\left\{\xi_{1}\left(\bar{\xi}_{1} \zeta\right)+\left(\bar{\zeta} \xi_{1}\right) \bar{\xi}_{2}\right\}=R(\zeta) 2 R\left(\xi_{1} \bar{\xi}_{2}\right) .
$$

$$
\xi_{1}\left(\bar{\xi}_{2} \zeta\right)=p \xi_{1} \eta_{2} \text { and }\left(\bar{\zeta} \xi_{1}\right) \bar{\xi}_{2}=p \bar{\eta}_{1} \bar{\xi}_{2} \text {. }
$$

Therefore, $p$ divides $2 R(\zeta) R\left(\xi_{1} \bar{\xi}_{2}\right)$. Since odd prime $p$ does not divide $\zeta$ in $J_{w}$ it does not divide $2 R(\zeta)$. Therefore, $p$ divides $R\left(\xi_{1} \bar{\xi}_{2}\right)$. But $R\left(\xi_{1} \xi_{2}\right)$ is an integer and $N\left(\xi_{1} \bar{\xi}_{2}\right)=p^{2}$. 
Now $\xi_{1} \neq \pm \xi_{2}$. Therefore $\xi_{1} \xi_{2} \neq p$. Hence $R\left(\xi_{1} \xi_{2}\right)=0$ and $\xi_{1} \xi_{2}$ has one or five odd rational integral components. Thus, as in the proof of Theorem $6, \xi_{1} \bar{\xi}_{2}$ is congruent modulo 2 in $J_{w}$ to a basic unit of $J_{0}$ other than \pm 1 . Therefore each element of $\xi$ of norm $p$ which divides $\zeta$ on the left in $J_{w}$ is congruent modulo 2 to a distinct element of norm 1 of $J_{w}$. Hence there exist at most 240 distinct factorizations $\xi \eta$ in this case. For all $\zeta$ of norm $p^{i+1}$ in $J_{w}$ the number of factorizations of this form is given by

$$
r_{w}(p) r_{w}\left(p^{l}\right) \text {. }
$$

Suppose that there exists a $\zeta$ in $J_{w}$ of norm $p^{l+1}$ which is not divisible by $p$ in $J_{w}$ with less than 240 factorizations of the type described. Then

$$
\begin{aligned}
r_{w}(p) r_{w}\left(p^{l}\right)< & 240 \sum_{N \zeta=p^{l+1}, p+\zeta} 1+240\left(1+p^{3}\right) \sum_{N \zeta=p^{l+1}, p \mid \zeta} 1 \\
= & 240\left\{r_{w}\left(p^{l+1}\right)-r_{w}\left(p^{l-1}\right)\right\} \\
& +240\left(1+p^{3}\right) r_{w}\left(p^{l-1}\right) \\
= & 240 r_{w}\left(p^{l+1}\right)+240 p^{3} r_{w}\left(p^{l-1}\right) \\
= & r_{w}(p) r_{w}\left(p^{l}\right) .
\end{aligned}
$$

Hence no such $\zeta$ exists. The result has thus been proved by contradiction. This completes the proof of Theorem 7 .

Let $\zeta$ be any element of an arithmetic $J_{h}$ for some $h\left(h=0,1, \ldots, 7, i_{1}, i_{2}, \ldots, i_{7}\right)$ of $C$. Suppose that $N \zeta=m n \neq 0$. We use a notation suggested by that of Rankin [10] and define

$$
\Omega_{h}(\zeta ; m, n)=\Omega_{h}(\zeta)
$$

to be the set of all factorizations $\xi \eta$ of $\zeta$ in $J_{h}$ for which $N \xi=m$ and $N \eta=n$. Also we define

$$
S_{h}(\zeta ; m, n)=S_{h}(\zeta)
$$

to be the number of such factorizations.

Thus we have proved, for $w$ a basic unit of $C$ other than 1, the following result.

TheOREM 6. For $m, n$ odd positive rational integers such that $(m, n)=1$,

$$
S_{w}(\zeta ; m, n)=240 \text {. }
$$

Also we have proved

THEOREM 7. For $p$ an odd rational prime and $l>0$

(i) if $p$ divides $\zeta$ in $J_{w}$

$$
S_{w}\left(\zeta ; p, p^{l}\right)=240\left(1+p^{3}\right), \quad \text { or }
$$

(ii) if $p$ does not divide $\zeta$ in $J_{w}$

$$
S_{w}\left(\zeta ; p, p^{\prime}\right)=240 .
$$

From Theorems 6 and 7 we deduce the following results.

THEOREM 8. For $m, n$ odd positive rational integers such that $(m, n)=1$

$$
S_{0}(\zeta ; m, n)=16 .
$$


THEOREM 9. For $p$ an odd rational prime and $l>0$

(i) if $p$ divides $\xi$ in $J_{0}$

$$
S_{0}\left(\zeta ; p, p^{\prime}\right)=16\left(1+p^{3}\right), \quad \text { or }
$$

(ii) if $p$ does not divide $\zeta$ in $J_{0}$

$$
S_{0}\left(\zeta ; p, p^{l}\right)=16
$$

THEOREM 10. For $m, n$ odd positive rational integers such that $(m, n)=1$ and any $t$ $(1 \leq t \leq 7)$

$$
S_{t}(\zeta ; m, n)=48
$$

THEOREM 11. For $p$ an odd rational prime, $l>0$, and any $t(1 \leq t \leq 7)$

(i) if $p$ divides $\zeta$ in $J_{t}$

$$
S_{t}\left(\zeta ; p, p^{\prime}\right)=48\left(1+p^{3}\right), \text { or }
$$

(ii) if $p$ does not divide $\zeta$ in $J_{t}$

$$
S_{t}\left(\zeta ; p, p^{\prime}\right)=48 \text {. }
$$

The methods used to establish Theorems 6 and 7 were first used by Rankin [10] to establish Theorems 8 and 9 above. We have used the idea of congruence modulo 2 in $J_{w}$, while Rankin used the fact that the eight basic units of $C$ are linearly independent and generate $J_{0}$ over the rational integers.

We have already see that $J_{0}$ occurs as a subset of $J_{w}$ for any basic unit $w$ of $C$ other than 1 and that $J_{t}(1 \leq t \leq 7)$ occus as a subset of $J_{w}$ for three basic unis $w$ of $C$ for which $w \neq 1$. Further, the elements of $J_{w}$ which belong to $J_{s}(0 \leq s \leq 7)$ are characterized as the elements of $J_{w}$ congruent modulo 2 in $J_{w}$ to an element of $J_{s}$ of norm 1. Thus Theorems 8 and 10 follow from Theorems 5 and 6 , while from Theorems 5 and 7 we deduce Theorem 9 and 11.

\section{REFERENCES}

1. F. van der Blij, History of the octaves, Wis- en Natuurkundig Tijdschrift (=Simon Stevin) 34, III (1961), 106-125.

2. F. Van der Blij and T. A. Springer, The arithmetics of the octaves and of the group $G_{2}$, Nederl. Akad. Wetensch. Proc. (=Indag. Math.) 62A (1959), 406-418.

3. P. J. C. Lamont, Arithmetics in Cayley's algebra, Proc. Glasgow Mathematical Assoc. 6 (1963), 99-106.

4. P. J. C. Lamont, Ideals in Cayley's algebra, Nederl. Akad. Wetensch. Proc. (=Indag. Math.) 66A (1963), 394-400.

5. P. J. C. Lamont, Approximation theorems for the group $G_{2}$, Nederl. Akad. Wetensch. Proc. (=Indag. Math.) 67A (1964), 187-192.

6. P. J. C. Lamont, Factorization and arithmetic functions for orders in composition algebras, Glasgow Mathematical Journal 14 (1973), 86-95.

7. P. J. C. Lamont, The number of Cayley integers of given norm, Proc. Edinburgh Math. Soc. 25 (1982), 101-103.

8. P. J. C. Lamont. Computer generated natural inner automorphisms of Cayley's algebra, Glasgow Mathematical Journal 23 (1982), 187-189.

9. P. J. C. Lamont, The nonexistence of a factorization formula for Cayley numbers, Glasgow Mathematical Journal 24 (1983), 131-132. 
10. R. A. Rankin, A certain class of multipicative functions, Duke Math. J. 13 (1946), 281-306.

11. O. Taussky, Sums of squares, Am. Math. Monthly 77 (1970), 805-830.

Department of Computer Science

College of ApPlied SCIENCES

WESTERN ILLINOIS UNIVERSITY

MACOMB, ILLINOIS 61455 